\title{
Finite-momentum condensation in a pumped microcavity
}

\author{
R. T. Brierley ${ }^{1}$ and P. R. Eastham ${ }^{1,2}$ \\ ${ }^{1}$ Cavendish Laboratory, University of Cambridge, Cambridge CB3 OHE, United Kingdom \\ ${ }^{2}$ School of Physics, Trinity College, Dublin 2, Ireland
}

(Received 7 December 2009; revised manuscript received 5 July 2010; published 28 July 2010)

\begin{abstract}
We calculate the absorption spectra of a semiconductor microcavity into which a nonequilibrium exciton population has been pumped. We predict strong peaks in the spectrum corresponding to collective modes analogous to the Cooper modes in superconductors and fermionic atomic gases. These modes can become unstable, leading to the formation of off-equilibrium quantum condensates. We calculate a phase diagram for condensation and show that the dominant instabilities can be at a finite momentum. Thus we predict the formation of inhomogeneous condensates, similar to Fulde-Ferrel-Larkin-Ovchinnikov states.
\end{abstract}

DOI: 10.1103/PhysRevB.82.035317 PACS number(s): 03.75.Kk, 71.36.+c, 71.35.Lk, 78.67.Hc

\section{INTRODUCTION}

The appearance of order at an equilibrium phase transition is a central concept in many areas of physics, from condensed matter to the physics of the early universe. Recently there has been considerable interest in the more general problem of ordering far from thermal equilibrium, motivated by the possibility of quantum quench experiments in cold atomic gases. ${ }^{1}$ In a quench the parameters of the system are rapidly switched from a disordered to an ordered phase and the disordered state forms the initial conditions for a dynamics with the new parameters. An interesting regime is that of coherent relaxationless dynamics, which can lead to the formation of nonequilibrium order including crystallization, ${ }^{2}$ condensation, and ferromagnetism. ${ }^{3}$

Among condensed matter systems, semiconductor microcavities are promising candidates for studying such quench dynamics. The nonequilibrium dynamics of microcavities has attracted considerable interest both experimentally ${ }^{4-9}$ and theoretically ${ }^{10-14}$ with recent experiments demonstrating regimes where the low-energy quasiparticles, polaritons, form a condensate. More recently, an experiment has been proposed to implement a quantum quench, ${ }^{15}$ by rapidly preparing a microcavity in a noncondensed initial state. The coherent dynamics of this noncondensed state is predicted to lead to a form of nonequilibrium condensation, similar to that predicted in a quenched Fermi gas. ${ }^{16-18}$ We show here that, as in the Fermi gas, such condensation is due to the appearance of a new collective mode. Moreover, we show that in the microcavity the dominant instability occurs at a finite wave vector. Thus we predict that microcavities could be used to realize inhomogeneous condensates, ${ }^{19}$ i.e., those characterized by a spatially varying phase. These condensates are similar, in this essential respect, to those predicted by Fulde, Ferrel, Larkin, and Ovchinnikov (FFLO) in unbalanced Fermi systems. ${ }^{19}$

In this paper, we first calculate the optical spectra of a microcavity a short time after it has been prepared in a noncondensed state, i.e., immediately after the "quench." We find that the collective mode responsible for condensation is directly observable in these spectra. We use this analysis to calculate a phase diagram for the nonequilibrium condensation and show that the condensation generally occurs at a finite momentum. While we focus on a microcavity containing quantum dots, our analysis is based on the MaxwellBloch equations. These describe a wide variety of coupled light-matter systems, implying a broad relevance of our work.

The remainder of this paper is structured as follows. In Sec. II we briefly review the proposed quench experiment and outline our model. In Sec. III we present absorption spectra of the system. In Sec. IV we discuss the phase diagram and the possibility of finite momentum condensation, and in Sec. V we discuss the connections to FFLO and the role of nonlinear terms. Section VI summarizes our conclusions. Finally, the appendix contains a brief treatment of the preparation of noncondensed initial states by optical pumping.

\section{MODEL}

We consider an experiment, proposed in Ref. 15, on a set of localized exciton states in a planar semiconductor microcavity. Such excitonic states could be realized in practice using either highly disordered quantum wells (where excitons are localized by disorder) or quantum dots. The proposed experiment involves two stages which are separated in time and can be regarded as independent. In the first stage, the localized states are driven by a chirped laser pulse. This pulse creates an energy-dependent population in the inhomogeneously broadened exciton line by adiabatic rapid passage (ARP). For certain populations a second stage may then occur, where the population evolves into a nonequilibrium condensate due to the photon-mediated interactions between the excitons.

As in Ref. 15 we describe the system using a generalization of the Dicke model. ${ }^{20}$ The localized exciton states are treated as two-level systems with the standard dipole coupling to the electromagnetic field. The state localized at site $i$ is specified by the Bloch vector $\sigma_{i}=\left\langle\hat{\sigma}_{i}\right\rangle$, where the inversion $\sigma_{i}^{z}=1(-1)$ for an occupied (unoccupied) state, and $\hat{\sigma}_{i}^{-}$is the exciton annihilation operator. Angle brackets \langle\rangle denote expectation values in the quantum state of the system.

We consider time scales short compared with the exciton lifetime, which is at least $100 \mathrm{ps}{ }^{21}$ and treat the electromagnetic field using a mean-field approximation. In this approxi- 
mation the photon creation and annihilation operators are replaced with their expectation values and hence become $c$ numbers. The resulting equations of motion are linear in the remaining operators so that we may take their expectation values without further approximation. The resulting dynamics obeys the generalized Maxwell-Bloch equations

$$
\begin{gathered}
i \dot{\psi}_{\mathbf{k}}=\omega_{\mathbf{k}} \psi_{\mathbf{k}}+g \int P_{\mathbf{k}} d E+f_{\mathbf{k}}+F \delta_{\mathbf{k}-\mathbf{p}}, \\
i \dot{P}_{\mathbf{k}}=E P_{\mathbf{k}}-g \sum_{\mathbf{k}^{\prime}} D_{\mathbf{k}-\mathbf{k}^{\prime}} \psi_{\mathbf{k}^{\prime}} \\
i \dot{D}_{\mathbf{k}}=2 g \sum_{\mathbf{k}^{\prime}}\left(P_{\mathbf{k}^{\prime}-\mathbf{k}}^{*} \psi_{\mathbf{k}^{\prime}}-P_{\mathbf{k}^{\prime}+\mathbf{k}} \psi_{\mathbf{k}^{\prime}}^{*}\right)
\end{gathered}
$$

Here $\psi_{\mathbf{k}}$ is the complex amplitude of the microcavity mode with in-plane wave vector $\mathbf{k}$ and energy $\omega_{\mathbf{k}}(\hbar=1)$. It is related to the expectation value of the photon annihilation operator by $\psi_{\mathbf{k}}=\left\langle\hat{\psi}_{\mathbf{k}}\right\rangle / \sqrt{N}$, where $N$ is the total number of localized states. This normalization is convenient when dealing with condensation since macroscopic occupation corresponds to a finite $\psi_{\mathbf{k}}$ in the thermodynamic limit $N \rightarrow \infty$. We allow for the finite lifetime of the photon modes by taking $\Im \omega_{\mathbf{k}}=-\gamma \cdot f_{\mathbf{k}}$ is introduced to allow us to calculate the linear response. $F$ is an externally applied pump field, with wave vector $\mathbf{p}$, that is used to create the nonequilibrium population.

The coupling $g=g_{i} \sqrt{n}$ in Eqs. (1)-(3) is related to the dipole coupling strengths of the localized states, $g_{i}$, and their area density $n$. To simplify the notation we have taken $g_{i}$ to be the same for all states; the extension to a distribution is straightforward. In the dipole gauge

$$
g_{i}=d \sqrt{\frac{E_{i}}{2 \epsilon_{0} \epsilon w}},
$$

where $d$ is the matrix element of the dipole operator $e \hat{r}$ between the zero-exciton and one-exciton states, and $E_{i}$ their energy difference. $w$ is the effective width of the cavity, which arises from the normalization of the cavity mode functions.

$P_{\mathbf{k}}(E)$ is the collective polarization of the ensemble at wave vector $\mathbf{k}$ due to states with energy in a small interval near $E$

$$
P_{\mathbf{k}}(E) \delta E=\frac{1}{N} \sum_{i}{ }^{\prime}\left\langle\hat{\sigma}_{i}^{-}\right\rangle e^{-i \mathbf{k} \cdot \mathbf{r}_{i}},
$$

where the prime indicates that the sum runs over states with exciton energies between $E$ and $\delta E . D_{\mathbf{k}}(E)$ is the collective inversion, defined in a similar way with $\hat{\sigma}^{z}$ replacing $\hat{\sigma}^{-}$.

In the following we shall be concerned with large $N$ and wave vectors which are small compared with the inverse spacing of the localized states. In these limits we may approximate sums over dot positions, such as those in Eq. (5), by

$$
\frac{1}{N} \sum_{i}{ }^{\prime} e^{-i \mathbf{k} \cdot \mathbf{r}_{i}} \approx \nu(E) \delta_{\mathbf{k}, 0} \delta E+O\left(\frac{1}{\sqrt{N}}\right) .
$$

Here $\nu(E)$ is the distribution of localized states in energy, normalized to one. Thus $N \nu(E) \delta E$ is the number of terms in the primed sum, Eq. (6). For $\mathbf{k} \neq 0$ the phasor sum is a twodimensional random walk, producing the $O(1 / \sqrt{N})$ corrections. $^{22}$

The approximation of Eq. (6) corresponds to replacing the response of the disordered dielectric with its homogeneous average response so that the wave vector is well-defined. This is similar to the linear dispersion model which has been extensively used for inorganic microcavities. ${ }^{23}$ The $O(1 / \sqrt{N})$ corrections describe Rayleigh scattering from density fluctuations in the dielectric. They are generally small corrections because the scatterers are dense so that on long wavelengths the medium appears homogeneous. The corrections can become important at very small or large wave vectors, ${ }^{24,25}$ for modes whose group velocity becomes very small. In this case a long lifetime is required for the wave vector to be well-defined so that even weak scattering or absorption destroys the quasipropagating modes. Here, however, we are concerned with modes that have a significant dispersion due to their photon component. Furthermore, the lifetime of these modes is massively enhanced by resonant gain from the populated excitons. Thus the leading approximation of Eq. (6) will capture the physics at the experimentally relevant wave vectors.

\section{ABSORPTION SPECTRA}

In the experiment $F$ is a chirped pulse, which creates a nonequilibrium population of excitons using ARP. In the Appendix we demonstrate this explicitly, using a model pulse, Eq. (A5), for which analytical solutions to the dynamics exist. Following the pulse the exciton states are populated with a distribution given by Eq. (A6) and the fields and polarizations are negligible $\psi_{\mathbf{k}} \approx 0, P_{\mathbf{k}} \approx 0$.

To establish the optical properties of the microcavity immediately after the pump pulse we find the response to a weak probe $f_{\mathbf{k}}$. The susceptibility can then be found from the induced electromagnetic field $\delta \psi_{\mathbf{k}} \equiv \Sigma_{\mathbf{k}^{\prime}} \int \chi_{\mathbf{k}, \mathbf{k}^{\prime}}(t$ $\left.-t^{\prime}\right) f_{\mathbf{k}^{\prime}}\left(t^{\prime}\right) d t^{\prime}$. If the system is stable then $\psi_{\mathbf{k}}$ and $P_{\mathbf{k}}$ are small (of order $f_{\mathbf{k}}$ ) for all times whereas if it is unstable they are only small soon after the pump pulse. In both regimes we may neglect terms above first order in $\psi_{\mathbf{k}}$ and $P_{\mathbf{k}}$. Equation (3) then gives $\dot{D}_{\mathbf{k}}=0$ so the nonequilibrium population is constant. Fourier transforming the linearized Eqs. (1) and (2) gives

$$
\omega \delta \psi_{\mathbf{k}}=\omega_{\mathbf{k}} \delta \psi_{\mathbf{k}}-g^{2} \sum_{\mathbf{k}^{\prime}} \int \frac{D_{\mathbf{k}-\mathbf{k}^{\prime}} \delta \psi_{\mathbf{k}^{\prime}}}{\omega-E} d E+f_{\mathbf{k}} .
$$

The pumping populates the states independently of their position, within the pump spot. Thus the sum in $D_{\mathbf{k}-\mathbf{k}^{\prime}}$ is strongly peaked near the forward scattering direction $\mathbf{k}-\mathbf{k}^{\prime}$ $=0$, as discussed above [Eq. (6)]. Neglecting the smaller offdiagonal scattering terms we obtain a diagonal response function 


$$
\chi_{\mathbf{k}}(\omega)=\frac{1}{\omega-\omega_{\mathbf{k}}+g^{2} \int \frac{D_{0}(E)}{\omega-E} d E} .
$$

The absorption coefficient of the microcavity follows from the susceptibility ${ }^{26,27}$

$$
A(\omega)=-2 \lim _{\epsilon \rightarrow 0} \operatorname{Im} \chi(\omega+i \epsilon),
$$

where the infinitesimal $\epsilon$ appears due to causality and can be physically understood as a small damping constant for the excitons. The sign is such that $A(\omega)>0$ corresponds to absorption of energy by the system. Thus from Eq. (8) we obtain

$$
\begin{aligned}
A(\omega) & =2 \frac{\gamma-g^{2} \pi D_{0}(\omega)}{\left(\omega-\omega_{c}+g^{2} \mathcal{P} \int \frac{D_{0}}{\omega-E} d E\right)^{2}+\left[\gamma-g^{2} \pi D_{0}(\omega)\right]^{2}} \\
& =2 \frac{H(\omega)}{G(\omega)^{2}+H(\omega)^{2}} .
\end{aligned}
$$

When the dots are unoccupied $D_{0}(\omega)<0$ and the empty exciton states contribute to absorption. For energies where there are occupied exciton states $D_{0}(\omega)>0$, describing gain due to the population.

In general, the response, Eq. (10), peaks near the zeroes of $G(\omega)$, which are at the energies of the normal modes. These energies differ from the energy of the cavity resonance due to the coupling to the exciton states. For an unpopulated state the condition $G(\omega)=0$ recovers the usual polariton energies of the Lorentz oscillator model ${ }^{28}$ but, in general, the spectrum differs due to the presence of the nonequilibrium population. The modes have a lifetime determined by the second factor in the denominator with contributions from the cavity losses and the resonant mixing with the band of exciton states. As expected it is damping which controls the overall strength of the absorption so that the damping factor $H(\omega)$ also appears in the numerator.

The only dependence of the spectra, Eqs. (8) and (10), on the wave vector is in the energy of the cavity mode, $\omega_{c}$ $=\mathfrak{R}\left(\omega_{\mathbf{k}}\right) \approx \omega_{0}+|\mathbf{k}|^{2} /(2 m)$. We therefore show results as functions of $\omega_{c}$, which corresponds experimentally to both the incident probe angle and the cavity width.

Figure 1 illustrates absorption spectra obtained from Eq. (10) for both a pumped and unpumped exciton line. These spectra are valid at all times if condensation does not occur (see later) but only soon after the pump pulse if it does. We have taken a Gaussian model for the inhomogeneously broadened exciton line with standard deviation $\sigma$, and measure energies relative to the center of the line. We choose the duration $\tau$ of the pump pulse as our unit of energy and have taken $g=13 / \tau, \gamma=1.5 / \tau, \sigma=15 / \tau$. These parameters, with $\tau$ $=3 \mathrm{ps}$, are reasonable for a microcavity containing strongly disordered quantum wells. ${ }^{29}$ As discussed in the appendix the pump creates a population equivalent to a Fermi function with temperature $1 /\left(\pi k_{B} \tau\right)$, and Fermi energy $\mu$ dictated by the chirp and center frequency of the pulse; we choose a pulse for which $\mu=-12.5 / \tau$.
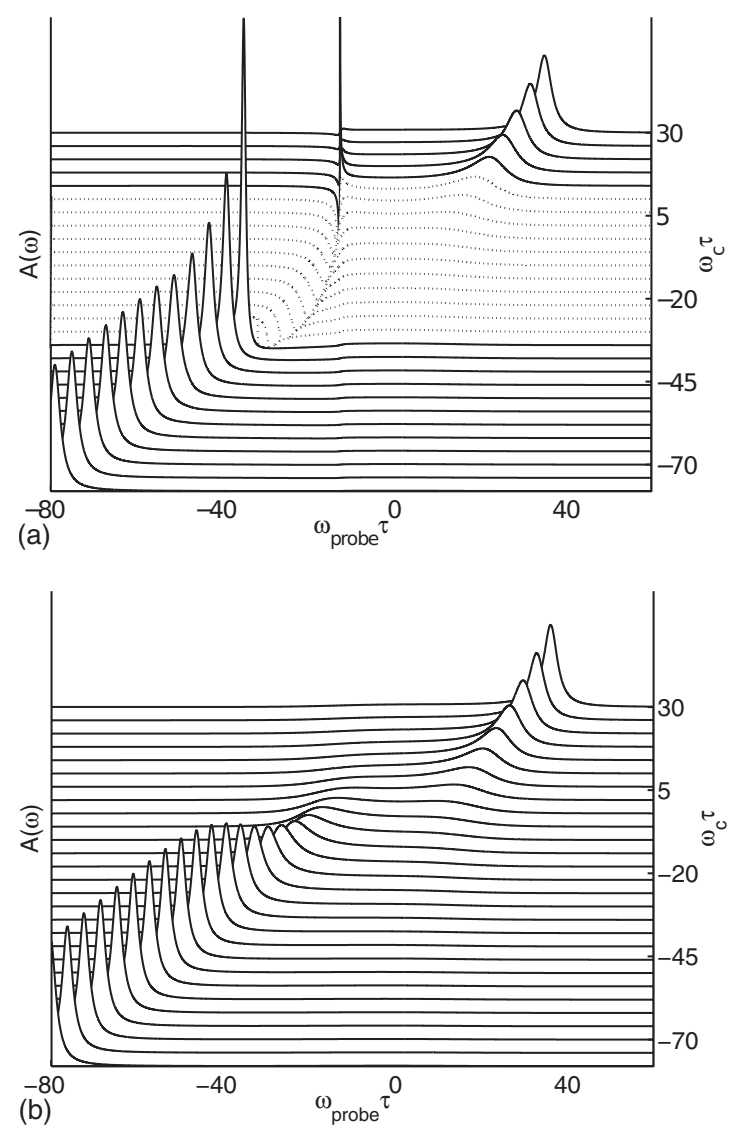

FIG. 1. Absorption spectra [Eq. (10)] in arbitrary units, for the parameters described in the text. Top panel: spectra with the exciton population created by the pump pulse. Bottom panel: spectra of the unpopulated microcavity. Each curve corresponds to a different value of the cavity mode energy $\omega_{c}$, vertically offset as indicated by the right-hand axes. Note the peak developing in the populated system at $\omega_{\text {probe }} \approx-13 / \tau$, indicating the presence of a collective mode. There are regions of probe gain $[A(\omega)<0]$ between -31 $<\omega_{\text {probe }} \tau<-13$, where gain from the populated excitons overcomes the cavity losses. Dotted lines indicate spectra with unstable normal modes. Instabilities occur when a normal mode lies in the region of gain and in these cases the absorption spectra have negative peaks, which are hidden from view in this figure.

The lower panel of Fig. 1 shows the expected result for an unpopulated microcavity. There is a pronounced peak in the absorption at the cavity mode energy, which broadens as the cavity mode is tuned through the excitons. There is some suggestion of an anticrossing near resonance, i.e., a polariton splitting but since the inhomogeneous broadening is relatively large compared with the coupling this is a weak effect. The top panel shows that the population dramatically changes the absorption spectrum. For these parameters it leads to a range of probe frequencies for which $\gamma$ $<g^{2} \pi D_{0}(\omega)$, and the absorption coefficient, Eq. (10), becomes negative. This occurs when the gain from the populated exciton states exceeds the losses so that there is a net gain for the probe beam. Moreover, we see a pronounced additional peak in the absorption spectrum, which first appears near the upper edge of the population as the cavity mode energy is decreased. As the cavity energy is decreased 
still further this peak moves down through the region of gain, before the spectrum finally reverts to one dominated by the unperturbed cavity mode.

This additional peak in the absorption spectrum is analogous to the Cooper pairing mode in a superconductor or Fermi gas, that gives rise to the Cooper instability. The analogy can be seen by noting that the normal-mode condition $G(\omega)=0$ contained in Eq. (10) is the Cooper equation, as discussed for this system in Ref. 15. The nonequilibrium exciton population corresponds to the Fermi distribution while the photon-mediated interaction between excitons corresponds to the pairing interaction between the electrons. As in a superconductor the sharp step in the population leads to collective modes generated by the pairing interaction. Figure 1 shows that, for reasonable parameters, these collective modes give rise to strong features in the spectra.

It is interesting to compare the spectra of Fig. 1 with the predictions for an equilibrium condensate in the same model. ${ }^{27}$ In that case the condensation opens a gap in the single-particle spectrum, which is the analog of the Cooper gap of the superconductor. Inside this gap is a collective mode, which is the analog of the Cooper mode or phase mode of the superconductor. The features visible in Fig. 1 arise from the nonequilibrium generalization of the collective mode (which is a different spectral feature than the gap). It is clear from Fig. 1 that it is the collective mode which dominates the spectrum. Thus, although the single-particle features may be affected by condensation, this would have little effect in practice. It may be possible to isolate the single particle spectrum in a Rayleigh scattering experiment, as has been proposed for equilibrium condensates. ${ }^{30}$

\section{PHASE DIAGRAM}

The normal modes of the system, with frequencies determined by $G(\omega)=0$, have decay rates $H(\omega)$. If a normal mode frequency lies in the $H(\omega)<0$ region produced by the nonequilibrium population it will be unstable, growing exponentially to give a state with a highly populated mode, i.e., a condensate. The condition for the onset of such an instability gives a nonequilibrium phase diagram, which is shown for our chosen parameters in Figs. 2 and 3.

Figure 2 shows the phase diagram assuming that only a single cavity mode, of energy $\omega_{c}$, is relevant. The dotted line shows the phase boundary for equilibrium condensation in the same model $^{27}$ with a temperature and chemical potential corresponding to the pumped population. We see that one sheet of the nonequilibrium phase boundary extends the equilibrium result to allow for the cavity damping. Whereas in equilibrium the presence of the collective mode is sufficient to create an instability, in the open system condensation only occurs if the gain at the energy of the collective mode overcomes the cavity loss. Thus the collective mode can exist even in the normal state (see Fig. 1) and the damping pushes the transition to larger couplings. In addition, we see that there is a lower limit on $\omega_{c}$ in Fig. 2. This lower threshold is a purely dynamical effect, not present in the equilibrium case. Below it there is a bosonic collective mode at an energy well below that of the populated states (see Fig. 1).

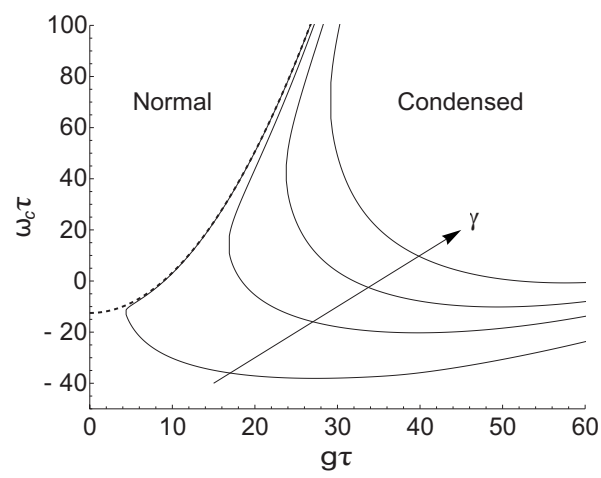

FIG. 2. Nonequilibrium phase diagram for a populated microcavity with a single photon mode as a function of coupling strength $g$ and cavity detuning $\omega_{c}$ with cavity damping $\gamma \tau$ $=1,15,30,45$. Arrow indicates curves of increasing $\gamma$. Dotted line indicates the location of the equilibrium phase boundary (Ref. 27) with temperature and chemical potential corresponding to that of the pumped population $\left[T=1 /\left(k_{B} \pi \tau\right), \mu=-12.5 / \tau\right]$.

Although this mode would be occupied in equilibrium it is far out of resonance with the excitons. As a result, it is not occupied dynamically and the uncondensed state is metastable. A similar metastable region has been predicted in quenched atomic gases. ${ }^{17}$

Figures 1 and 2 show that, for a given $g$ and $\gamma$, the condensation instability occurs over a range of $\omega_{c}$. In a microcavity different values of $\omega_{c}=\omega_{0}+|\mathbf{k}|^{2} / 2 m$ correspond to either changing the cavity width, which varies the detuning $\omega_{0}$, or considering modes at a different wave vector $\mathbf{k}$. As such, a range of unstable $\omega_{c}$ implies that for a fixed cavity detuning there can be instabilities at many wave vectors with different growth exponents $|H(\omega)|$. At short times after the population has been created the mode with the highest growth exponent will dominate. Figure 4 shows that as $\omega_{0}$ is lowered this dominant mode occurs at $\mathbf{k} \neq 0$, implying a condensate with finite momentum, and a spatially inhomogeneous order parameter. Thus the full phase diagram, allowing for the continuum of in-plane modes, takes the form shown in Fig. 3.

The phase diagram of Fig. 3 can be understood physically by noting that the condensation is a result of the exciton-

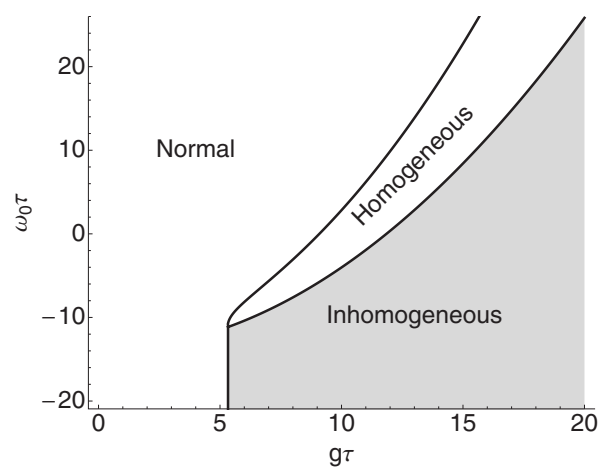

FIG. 3. Nonequilibrium phase diagram for a populated microcavity with a continuum of in-plane photon modes as a function of the coupling constant $g$ and detuning at $\mathbf{k}=0, \omega_{0}$. The cavity damping $\gamma=1.5 / \tau$. In the inhomogeneous region modes with $\mathbf{k} \neq 0$ have the highest growth exponent. The vertical boundary is a result of the gain-loss criterion, $H(\omega)>0$. 


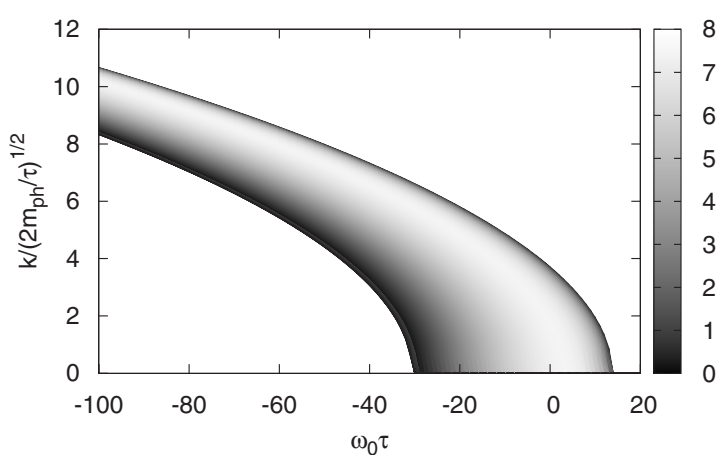

FIG. 4. Growth exponents $-H(\omega)$ of the unstable modes with wave vector modulus $k$ in a populated cavity with detuning $\omega_{0}$, coupling strength $g=13 / \tau$, and decay constant $\gamma=1.5 / \tau$. The wave vector of the most unstable mode increases as $\omega_{0}$ is reduced. For $\omega_{c} \lesssim-30 / \tau$ the $\mathbf{k}=0$ mode is stable and there are only finite momentum instabilities.

photon interactions. If the cavity mode is detuned well below the excitons then the quasiparticles at $\mathbf{k}=0$ are essentially photons, uncoupled from the excitons. Thus the condensation shifts to the higher momentum states, where the photons and excitons are closer to resonance, and there are strong coupling effects.

\section{DISCUSSION}

There has been extensive theoretical work on states with finite momentum Cooper pairing in the context of equilibrium superconductors, atomic gases, and quark matter. ${ }^{19}$ These FFLO states, which have been sought in a wide range of systems, may be the ground states where there is an imbalance in the populations of the two pairing species. However, they involve increasing the kinetic energy in order to gain pairing energy and in practice this restricts them to small regions of parameter space. Here, however, the state achieved is determined by the Cooper equation and a gainloss criterion with the energetics playing a subsidiary role. Thus, as indicated by Fig. 3, condensation at a finite momentum may be achieved without fine tuning of parameters.

The connection to FFLO may be made more explicit using a representation for the exciton operator $\hat{\sigma}_{i}^{-}=\hat{c}_{i, \uparrow} \hat{c}_{i, \downarrow}$, where $\hat{c}$ is a fermionic annihilation operator. In the simplest case of a plane wave condensate at wave vector $\mathbf{k}$ the meanfield order parameter is $P_{\mathrm{k}}$, the macroscopic component of the exciton polarization. This becomes

$$
\begin{aligned}
P_{\mathbf{k}} & =(1 / N) \sum_{i}\left\langle\hat{c}_{i, \uparrow} \hat{c}_{i, \downarrow}\right\rangle e^{-i \mathbf{k} \cdot \mathbf{r}_{i}} \\
& =1 / N^{2} \sum_{\mathbf{p}, \mathbf{q}}\left\langle\hat{c}_{\mathbf{p}, \uparrow} \hat{c}_{\mathbf{q}, \downarrow}\right\rangle \sum_{i} e^{i(\mathbf{p}+\mathbf{q}) \cdot \mathbf{r}_{i}-i \mathbf{k} \cdot \mathbf{r}_{i}} \\
& \sim(1 / N) \sum_{\mathbf{p}}\left\langle\hat{c}_{\mathbf{p}, \uparrow} \hat{c}_{-\mathbf{p}-\mathbf{k}, \downarrow}\right\rangle .
\end{aligned}
$$

Thus we see that the condensate can be formally represented as a coherent state of fermions, pairing with a finite total momentum. While in this respect the state is similar to FFLO, there are other differences. For example, in Eq. (11) the relative wave function of the pair is independent of momentum and the pairing is entirely local. In a general FFLO state there is a momentum-dependent pairing function, describing Cooper pairs of finite size.

Because the growth exponent depends only on $|\mathbf{k}|$ the condensate emission at short times will cover a circle of in-plane wave vectors, giving a cone of emitted light. However at later times the nonlinear terms neglected in Eq. (7) will break the degeneracy, selecting a spatial form for the condensate. In equilibrium such interactions favor condensate structures consisting of a pair of antipodal wave vectors $(\mathbf{k},-\mathbf{k})$, or more complex structures such as face-centered cubes. ${ }^{19}$ Here the nonlinearity corresponds to the depletion of the exciton population by the growth of the condensate. This will reduce the gain ${ }^{13}$ for collective modes of similar energies, suggesting that a single plane wave (Fulde-Ferrel) state may be favored. Although these nonlinearities determine a particular form for the condensate it is unlikely they will lead to a homogeneous state so we do not treat them in detail here.

It is interesting to note that finite-momentum polariton condensates have been observed ${ }^{4,5,9}$ though in a different experimental protocol to that considered here. In these cases there is continuous pumping and relaxation and a spatial structure imposed by a pump and trap. The mechanisms leading to this finite momentum condensate have yet to be established and are likely different from those discussed here. Nonetheless, these experiments demonstrate that microcavities could support exotic ordered states that have proved elusive in equilibrium.

\section{SUMMARY}

We have calculated the linear response of a microcavity with a nonequilibrium population of excitons. The population produces new collective modes, which are analogs of the Cooper pairing mode in superconductors. We have shown that these modes are visible as peaks in the optical spectra. By considering the growth exponents of these collective modes we have found a phase diagram for the dynamical condensation. In a microcavity with a continuum of in-plane wave vectors there can be multiple unstable modes of different wave vectors. For some parameters the dominant (and, for sufficiently negative detuning, only) instabilities can occur at a nonzero wave vector. In these regimes the microcavity will develop a condensate with spatial structure, signaled by coherent emission at an angle to the cavity normal.

\section{ACKNOWLEDGMENTS}

This work was supported by Science Foundation Ireland under Grant No. 09/SIRG/I1592 and EPSRC under Grant No. EP/F040075/1. We thank P. B. Littlewood, J. Keeling, and M. Parish for discussions and comments on the manuscript.

\section{APPENDIX: ANALYTICAL PUMP SOLUTION}

Reference 15 gives the results of numerical simulations of Eqs. (1)-(3), driven by a linearly chirped Gaussian pump 
pulse. These simulations show that there are parameter regimes in which the dynamics separates into a fast pumping stage, followed by a slower condensation stage. In this appendix we present an approximate analytical solution to Eqs. (1)-(3) which gives the population and (negligible) polarization at the end of the pump pulse. This solution forms the starting point for the dynamics discussed in the body of the paper.

The full numerical solutions in Ref. 15 show that the only significant polarization during the pumping is at the pump wave vector p. Moreover, this polarization can be seen to be small compared with the applied pump field $F$. Thus during the pumping we may neglect the second term in Eq. (1) for all wave vectors. With this approximation, Eqs. (1)-(3) reduce to an ensemble of independent two-level systems, driven by a field $\psi_{\mathbf{p}}^{0}$ which is the externally applied field $F$ filtered by the cavity response. For pumping at high angles, outside the stop band of the mirrors, $\psi_{\mathbf{p}}^{0}$ is proportional to the pump pulse. Thus Eqs. (1)-(3) become the Bloch equations

$$
\left(\begin{array}{c}
\dot{P}_{x}^{\prime} \\
\dot{P}_{y}^{\prime} \\
\dot{D}_{0}
\end{array}\right)=\left(\begin{array}{ccc}
0 & E-\Delta(t) & 0 \\
-E+\Delta(t) & 0 & g \Omega(t) \\
0 & -g \Omega(t) & 0
\end{array}\right)\left(\begin{array}{c}
P_{x}^{\prime} \\
P_{y}^{\prime} \\
D_{0}
\end{array}\right),
$$

where we have defined

$$
\begin{gathered}
\psi_{\mathbf{p}}^{0}=\Omega(t) e^{-i \int^{t} \Delta(t) d t^{\prime}}, \\
P^{\prime}=P_{\mathbf{p}} e^{i \int^{t} \Delta(t) d t^{\prime}}, \\
P^{\prime}=\frac{1}{2}\left(P_{x}-i P_{y}\right) .
\end{gathered}
$$

Note that the collective polarizations are at the pump wave vector while the collective inversion is spatially uniform.

For a model pump pulse of the form

$$
g \Omega(t)=\frac{\Omega_{0}}{\tau} \operatorname{sech} \frac{t-t_{0}}{\tau},
$$

$$
\Delta(t)=\frac{\alpha}{\tau} \tanh \frac{t-t_{0}}{\tau}+\nu_{0},
$$

Equation (A1) has an analytical solution. ${ }^{31}$ The form of the population $D_{0}$ at times $t \gg \tau$ after the pulse is

$$
\begin{aligned}
\frac{D_{0}(E)}{\nu(E)}= & 2 \frac{\cosh ^{2} \frac{\pi \alpha}{2}-\cos ^{2} \frac{\pi \sqrt{\Omega_{0}^{2}-\alpha^{2}}}{2}}{\cosh \left\{\frac{\pi}{2}\left[\left(E-\nu_{0}\right) \tau-\alpha\right]\right\} \cosh \left\{\frac{\pi}{2}\left[\left(E-\nu_{0}\right) \tau+\alpha\right]\right\}} \\
& -1 .
\end{aligned}
$$

In the limit $\Omega_{0}>\alpha \gg 1$ the distribution becomes

$$
\frac{D_{0}(E)}{\nu(E)}=2 n_{F}\left(E-\mu_{+}-\nu_{0}\right)\left[1-n_{F}\left(E-\mu_{-}-\nu_{0}\right)\right]-1,
$$

where $n_{F}(E)$ is a Fermi distribution with temperature $1 /\left(k_{B} \pi \tau\right)$ and the chemical potentials $\mu_{ \pm}=\nu_{0} \pm \frac{\alpha}{\tau}$. If the density of states $\nu(E)$ is sufficiently small at energies below $\mu_{-}$ then this lower edge is irrelevant. The occupation function $D_{0}(E)$ is then equivalent to an equilibrium Fermi distribution with $\mu=\mu_{+}$. In this paper we consider parameters where this applies, choosing $\nu_{0}=-30 / \tau, \Omega_{0}=18$, and $\alpha=17.5$.

Since the dynamics during the pumping, Eq. (A1), involves only $P_{\mathrm{p}}$, the polarization at any other wave vector $P_{\mathbf{k} \neq \mathbf{p}}$ remains zero. For the polarization at the pump wave vector, the analytical solution gives a window of energies $\sim \tau$ in which there is a nonzero polarization after pumping. However, in the absence of an external field and with $\psi_{\mathbf{p}} \approx 0$, as is the case after pumping, the subsequent evolution of the polarization is free. As a result, the total polarization $P_{\mathrm{p}}$ $=\int P_{\mathbf{p}}(E) d E$ decays by free induction decay and so may be neglected after a time of order $\tau$. The numerical work of Ref. 15 showed that for suitable parameters the preparation of this state, including the free induction decay of the remnant polarization, finishes before the dynamics discussed in the main body of this paper takes place.
${ }^{1}$ I. Bloch, J. Dalibard, and W. Zwerger, Rev. Mod. Phys. 80, 885 (2008).

${ }^{2}$ C. Kollath, A. M. Läuchli, and E. Altman, Phys. Rev. Lett. 98, 180601 (2007).

${ }^{3}$ M. Babadi, D. Pekker, R. Sensarma, A. Georges, and E. Demler, arXiv:0908.3483 (unpublished).

${ }^{4}$ M. Richard, J. Kasprzak, R. Romestain, R. André, and L. S. Dang, Phys. Rev. Lett. 94, 187401 (2005).

${ }^{5}$ C. W. Lai et al., Nature (London) 450, 529 (2007).

${ }^{6}$ J. Kasprzak, D. D. Solnyshkov, R. André, L. S. Dang, and G. Malpuech, Phys. Rev. Lett. 101, 146404 (2008).

${ }^{7}$ M. Maragkou, A. J. D. Grundy, E. Wertz, A. Lemaître, I. Sagnes, P. Senellart, J. Bloch, and P. Lagoudakis, Phys. Rev. B 81, 081307(R) (2010).
${ }^{8}$ D. Sanvitto, A. Amo, L. Viña, R. André, D. Solnyshkov, and G. Malpuech, Phys. Rev. B 80, 045301 (2009).

${ }^{9}$ D. N. Krizhanovskii et al., Phys. Rev. B 80, 045317 (2009).

${ }^{10}$ M. H. Szymańska, J. Keeling, and P. B. Littlewood, Phys. Rev. B 75, 195331 (2007).

${ }^{11}$ M. Wouters and I. Carusotto, Phys. Rev. Lett. 99, 140402 (2007).

12 J. Keeling and N. G. Berloff, Phys. Rev. Lett. 100, 250401 (2008).

${ }^{13}$ P. R. Eastham, Phys. Rev. B 78, 035319 (2008).

${ }^{14}$ T. D. Doan, H. T. Cao, D. B. Tran Thoai, and H. Haug, Phys. Rev. B 72, 085301 (2005).

${ }^{15}$ P. R. Eastham and R. T. Phillips, Phys. Rev. B 79, 165303 (2009). 
${ }^{16}$ A. V. Andreev, V. Gurarie, and L. Radzihovsky, Phys. Rev. Lett. 93, 130402 (2004).

${ }^{17}$ R. A. Barankov and L. S. Levitov, Phys. Rev. Lett. 93, 130403 (2004).

${ }^{18}$ E. A. Yuzbashyan, O. Tsyplyatyev, and B. L. Altshuler, Phys. Rev. Lett. 96, 097005 (2006).

${ }^{19}$ R. Casalbuoni and G. Nardulli, Rev. Mod. Phys. 76, 263 (2004).

${ }^{20}$ J. Keeling, P. R. Eastham, M. H. Szymańska, and P. B. Littlewood, Phys. Rev. B 72, 115320 (2005).

${ }^{21}$ W. Langbein and B. Patton, Phys. Rev. Lett. 95, 017403 (2005).

${ }^{22}$ J. W. Goodman, in Laser Speckle and Related Phenomena, edited by J. C. Dainty (Springer, Berlin, 1975).

${ }^{23}$ V. Savona, J. Phys.: Condens. Matter 19, 295208 (2007).

${ }^{24}$ V. M. Agranovich, M. Litinskaia, and D. G. Lidzey, Phys. Rev. B
67, 085311 (2003).

${ }^{25}$ M. Litinskaya and P. Reineker, Phys. Rev. B 74, 165320 (2006).

${ }^{26}$ G. D. Mahan, Many-Particle Physics, 2nd ed. (Springer, 1990).

${ }^{27}$ P. R. Eastham and P. B. Littlewood, Phys. Rev. B 64, 235101 (2001).

${ }^{28}$ G. Burns, Solid State Physics (Academic, London, 1985), Chap. 13.

${ }^{29}$ I. M. Piper, P. R. Eastham, M. Ediger, A. M. Wilson, Y. Wu, M. Hugues, M. Hopkinson, and R. T. Phillips, J. Phys.: Conf. Ser. (to be published); arXiv:1007.1171.

${ }^{30}$ F. M. Marchetti, J. Keeling, M. H. Szymanska, and P. B. Littlewood, Phys. Rev. B 76, 115326 (2007).

${ }^{31}$ F. T. Hioe and C. E. Carroll, Phys. Rev. A 32, 1541 (1985). 